\title{
Phyto-oestrogens and colorectal cancer risk: a systematic review and dose-response meta-analysis of observational studies
}

\author{
Ruijingfang Jiang ${ }^{1}$, Akke Botma ${ }^{1}$, Anja Rudolph ${ }^{1}$, Anika Hüsing ${ }^{1}$ and Jenny Chang-Claude ${ }^{1,2_{*}}$ \\ ${ }^{1}$ Division of Cancer Epidemiology, German Cancer Research Center (DKFZ), 69120 Heidelberg, Germany \\ ${ }^{2}$ University Cancer Center Hamburg (UCCH), University Medical Center Hamburg-Eppendorf, 20246 Hamburg, Germany \\ (Submitted 12 August 2016 - Final revision received 16 November 2016 - Accepted 26 November 2016)
}

\section{Abstract}

Epidemiological studies suggest that soya consumption as a source of phyto-oestrogens and isoflavones may be associated with a reduced risk of colorectal cancer. However, findings have not yet been synthesised for all groups of phyto-oestrogens. A meta-analysis was conducted to quantify the association between phyto-oestrogens and colorectal cancer risk. Relevant observational studies published up to June 2016 were identified by searching MEDLINE, EMBASE and Cochrane Library databases. Study-specific relative risks (RR) were pooled in both categorical and dose-response meta-analyses. Out of seventeen identified studies, sixteen were included in the meta-analysis. Comparing the highest with the lowest intake category, inverse associations for phyto-oestrogens overall and by subgroup were observed but were statistically significant in case-controls studies and not in cohort studies. The pooled RR in case-control studies were 0.76 (95\% CI 0.69, 0.84), 0.77 (95\% CI $0.69,0.85)$ and $0.70(95 \%$ CI $0.56,0.89)$ for phyto-oestrogens, isoflavones and lignans, respectively, whereas the corresponding pooled RR were 0.95 (95\% CI 0.85, 1.06), 0.94 (95\% CI 0.84, 1.05) and 1.00 (95\% CI 0.64, 1.57) in cohort studies. Dose-response analysis yielded an $8 \%$ reduced risk of colorectal neoplasms for every $20 \mathrm{mg} / \mathrm{d}$ increase in isoflavones intake in Asians (pooled RR 0.92; 95\% CI 0.86, 0.97). A nonlinear inverse association with colorectal cancer risk was found for lignans intake, but no association for circulating enterolactone concentrations was observed. Thus, study heterogeneity precludes a rigorous conclusion regarding an effect of high exposure to isoflavones on risk of colorectal cancer. Current evidence for an association with lignans exposure is limited. Further prospective studies, particularly evaluating lignans, are warranted to clarify the association between different phyto-oestrogens and colorectal cancer risk.

Key words: Phyto-oestrogens: Isoflavones: Lignans: Colorectal cancer: Meta-analyses

Phyto-oestrogens have been postulated to lower the risk of colorectal cancer on the basis of the fact that the incidence of colorectal cancer is lower in populations with a high intake of phyto-oestrogens $^{(1,2)}$. Phyto-oestrogens are plant-derived heterocyclic phenols and have similar structural features as endogenous oestrogens, which enable them to bind oestrogen receptors ${ }^{(3,4)}$. The activation of oestrogen receptors by phyto-oestrogens may induce the transcription of genes involved in angiogenesis, cellular adhesion, proliferation and apoptosis ${ }^{(5)}$. These altered transcriptional activities are likely to be involved in the anticarcinogenic effects against colorectal cancer, but the exact protective mechanisms remain to be elucidated. There are three principal groups of phyto-oestrogens: isoflavones, lignans and coumestans $^{(6,7)}$. Isoflavones, mainly found in soyabeans, are the most common phyto-oestrogens in Asian populations, as soya foods are regularly consumed in Asian countries. In Western populations, the predominant source of phyto-oestrogens are lignans, which are present in seeds, grains, vegetables, fruits and animal products ${ }^{(8)}$. Coumestans account for $<5 \%$ of total phyto-oestrogen intake ${ }^{(9)}$, and are mainly found in soyabean sprout and spinach. To become biologically active, the inactive plant-lignan precursors must be metabolised by the gut microflora into mammalian lignans - namely, enterodiol and enterolactone $^{(10,11)}$. Isoflavones are more bioactive than lignans, especially in the form of aglycone from soyabean-fermented products, which can be absorbed directly by human intestines ${ }^{(12)}$.

Earlier epidemiological studies have predominantly studied soya consumption in relation to colorectal cancer ${ }^{(13,14)}$. More recent studies have specifically assessed the dietary intake of isoflavones, the bioactive constituent in soya. These studies reported inconsistent associations between isoflavone exposure and colorectal cancer risk, including inverse associations ${ }^{(15-19)}$, positive associations $^{(20)}$ as well as null associations ${ }^{(21-28)}$. A recent meta-analysis found that high isoflavone intake was associated with a $24 \%$ lower risk of colorectal cancer ${ }^{(29)}$. However, no published meta-analyses to date have assessed the association of colorectal cancer risk with other phyto-oestrogen subtypes and their potential dose-response relationships.

\footnotetext{
Abbreviations: ESR2, oestrogen receptor $\beta$; RR, relative risk.
}

* Corresponding author: Professor, Dr J. Chang-Claude, fax +49 6221 422203, email j.chang-claude@dkfz-heidelberg.de 
The aims of this meta-analysis were therefore to summarise the current evidence on exposure to phyto-oestrogens in relation to the risk of colorectal cancer and adenoma and to provide a comprehensive review of the associations found with the two main classes of phyto-oestrogens - isoflavones and lignans. We further conducted a dose-response analysis and explored sources of heterogeneity among studies.

\section{Methods \\ Search strategy}

This study was conducted and reported according to the Preferred Reporting Items for Systematic Reviews and MetaAnalyses statement ${ }^{(30)}$. We carried out a literature search in MEDLINE (from 1975), EMBASE (from 1984) and Cochrane Library (from 1993) databases up to June 2016 without language restrictions to identify observational studies (case-control and cohort studies) that examined the association between phytooestrogens and risk of colorectal, colon or rectal neoplasms (cancer or adenoma). The following terms were used as search strategy: '(colorectal OR colon OR rectum) AND (cancer OR tumor OR neoplasm OR malignancy OR adenoma) AND (phytoestrogen OR isoflavone OR lignan)'. The detailed search strategies are available in the online Supplementary Material. Furthermore, we manually reviewed references of relevant articles to identify additional articles. There are no clinical trials on this topic.

\section{Study selection}

After removal of duplicate articles across databases, two independent investigators (R. J. and A. B.) conducted an initial screening on titles and abstracts to remove clearly irrelevant publications such as editorials, reviews, experimental studies or those not reporting relevant exposures or outcomes. Full-texts of potential publications were then reviewed by the same investigators to identify eligible studies, which reported relative risks (RR) (hazard ratio, RR or odds ratio) for the association between risk of colorectal cancer or adenoma and phytooestrogens, measured as either dietary consumption or circulating biomarkers.

\section{Data extraction}

Two investigators (R. J. and A. B.) independently reviewed the eligible studies and extracted data, and accuracy was checked thereafter. The following data were extracted from the identified publications: author, year of publication, region of the study, study period, sex and age of participants, study type, number of cases and non-cases, method of exposure assessment, type of phyto-oestrogens, exposure level, outcome ascertainment, covariates in the fully adjusted models, and RR and corresponding $95 \%$ CI for the most fully adjusted models. The quality of each study was assessed using the NewcastleOttawa scale (NOS) based on participant selection, exposure and outcome ascertainment, as well as potential confounding ${ }^{(31)}$ (online Supplementary Material). The NOS ranged from 0 to a maximum of 9 , and a higher score is indicative of higher study quality. For studies with missing information, attempts were made to contact the corresponding author of the original studies for further information.

\section{Statistical analysis}

Meta-analyses were conducted for overall phyto-oestrogens (isoflavones and lignans combined) and separately for isoflavones and lignans. We used the RR from the highest $v$. the lowest category of exposure in the meta-analysis, as exposure assessment methods and categorisation differed among studies. In the meta-analysis for overall phyto-oestrogens, when a study reported RR for the specific type(s) of phyto-oestrogens, but not for overall phyto-oestrogens, we used the RR for the only reported subtype (isoflavones or lignans) or the most commonly consumed subtype in that population. Therefore, for a Swedish study that reported estimates for dietary isoflavones and lignans separately, only the RR for lignans, the major consumed phytooestrogen, were included ${ }^{(28)}$. Combined RR and 95\% CI were computed in the meta-analyses using a random-effect model when between-study heterogeneity was observed $(P<0 \cdot 1)$, whereas the fixed-effect model was used otherwise. All analyses were conducted both for colorectal cancer and for colorectal neoplasms and separately for cohort (including nested casecontrol and case-cohort studies) and case-control studies.

Dose-response analysis was carried out for dietary isoflavones intake, lignans intake and circulating enterolactone concentrations only, because of limited studies on circulating isoflavone concentrations ${ }^{(27)}$. We used the method proposed by Greenland \& Longnecker ${ }^{(32)}$ under random-effect model.

For the dose-response analysis of isoflavones and lignans, units were transformed to $\mathrm{mg} / \mathrm{d}$ for all eligible studies. For the Spanish study ${ }^{(20)}$, reported intakes (mg/4184 kJ (mg/1000 kcal)) were multiplied by 2 to account for the average energy intake of $8368 \mathrm{~kJ} / \mathrm{d}(2000 \mathrm{kcal} / \mathrm{d})$ in the study population. For the Swedish study ${ }^{(28)}$, reported intakes $\mu \mathrm{g} /(\mathrm{d} \times \mathrm{MJ})$ were multiplied by 6.5 to account for the average energy intake of $6500 \mathrm{~kJ} / \mathrm{d}$ in the study population. To derive a dose-response trend for each study, the distribution of cases and non-cases, RR and corresponding CI as well as cut-off values for at least three categories were required. If $\mathrm{RR}$ were available only for male or female separately, separate dose-response curves were derived and combined using fixed-effect models. Median or mean levels of isoflavones intake for each category were assigned to the corresponding RR. When these were not reported, the midpoint of the upper and lower boundaries was used. For studies with an unbounded highest category, we assumed the width of that category to be the same as its closest adjacent category. For studies that used an unbounded lowest category, the lower boundary was set to $0 \mathrm{mg} / \mathrm{d}$. As isoflavones intake varies greatly between Asian and Western populations, dose-response analyses were conducted separately for Asian and Western studies. A potential, non-linear dose-response relationship was examined by using restricted cubic splines with three fixed percentiles (10, 50 and 90\%) and testing whether the coefficient of the second spline equals $0^{(33,34)}$. Given the scarce number of studies available, case-control and cohort studies were combined for the dose-response analysis of dietary isoflavones as 
well as lignans. However, analysis according to study design was performed, where possible.

For the dose-response analysis of the biomarker enterolactone, we converted all RR corresponding to per doubling in enterolactone concentration after applying a $\log _{2}$ transformation to the exposure level. For the two Dutch studies ${ }^{(35,36)}$, which used $\log _{10}$ transformation and an increment of $\log _{10}$ 14.61 and $\log _{10} 39 \cdot 1$, we consequently adjusted the RR and corresponding CI to the power of $0.26\left(\log _{10} 2 / \log _{10} 14 \cdot 61\right)$ and $0 \cdot 19\left(\log _{10} 2 / \log _{10} 39 \cdot 1\right)$.

Heterogeneity was evaluated by Cochran's $Q$ test, and the proportion of total variation due to heterogeneity was quantified by $I^{2(37)}$. For the Cochran's $Q$ test, we defined statistical significance as $P<0.1$ rather than the conventional level of $P<0.05$, because of the low power of this test ${ }^{(38)}$. To explore heterogeneity across the studies, we performed a sensitivity analysis for overall phyto-oestrogen meta-analysis by leaving one study out at a time and calculated the pooled RR in the remaining studies. We further conducted subgroup analysis by phyto-oestrogen subtype, study design, ethnicity, cancer site and sex to explore the sources of heterogeneity. To assess possible bias due to confounding, we conducted pooled analyses stratified by adjustment factors, including BMI, physical activity, total energy intake, family history of colorectal cancer and non-steroidal anti-inflammatory drug (NSAID) use. Egger's regression test and funnel plots were used to evaluate small study effects ${ }^{(39)}$. Apart from what was specified, the significance level was set to 0.05 in all the analyses. All analyses were carried out using the metaphor ${ }^{(40)}$ and dosresmeta ${ }^{(41)}$ packages in $\mathrm{R}$ environment (version 3.1.2).

\section{Results}

\section{Literature search}

We identified twenty-three potential publications of twenty studies for full-text assessment ${ }^{(15-28,35,36,42-48)}$, of which four ${ }^{(43,45,46,48)}$ were excluded and two could only be included for narrative review ${ }^{(22,42)}$ (Fig. 1). Two of the excluded studies did not provide RR for relevant exposures ${ }^{(43,45)}$, one was a duplicate report ${ }^{(46)}$ of an included study ${ }^{(18)}$ and the other one was a recent conference abstract $^{(48)}$ of an included study ${ }^{(24)}$. The two studies ${ }^{(22,27,42)}$ that could be reviewed narratively found null associations between phyto-oestrogens intake ${ }^{(22)}$, isoflavones intake ${ }^{(22,42)}$ or isoflavone concentration $^{(27)}$ and colorectal cancer risk. Enterolignans intake was found to be associated with a reduced risk of colorectal cancer in females but not in males ${ }^{(22)}$. Among these two studies that could not be included, one study did not provide a $\mathrm{RR}^{(42)}$ and the other study reported a RR per doubling of phyto-oestrogen, isoflavones and enterolignans intake ${ }^{(22)}$ and isoflavones concentration $^{(27)}$, which could not be transformed to highest $v$. lowest $R R$ because the distribution of phyto-oestrogen intake or

1179 studies identified through initial literature search

- PubMed: $n 494$

- Embase: $n 684$

- Cochrane: $n 1$

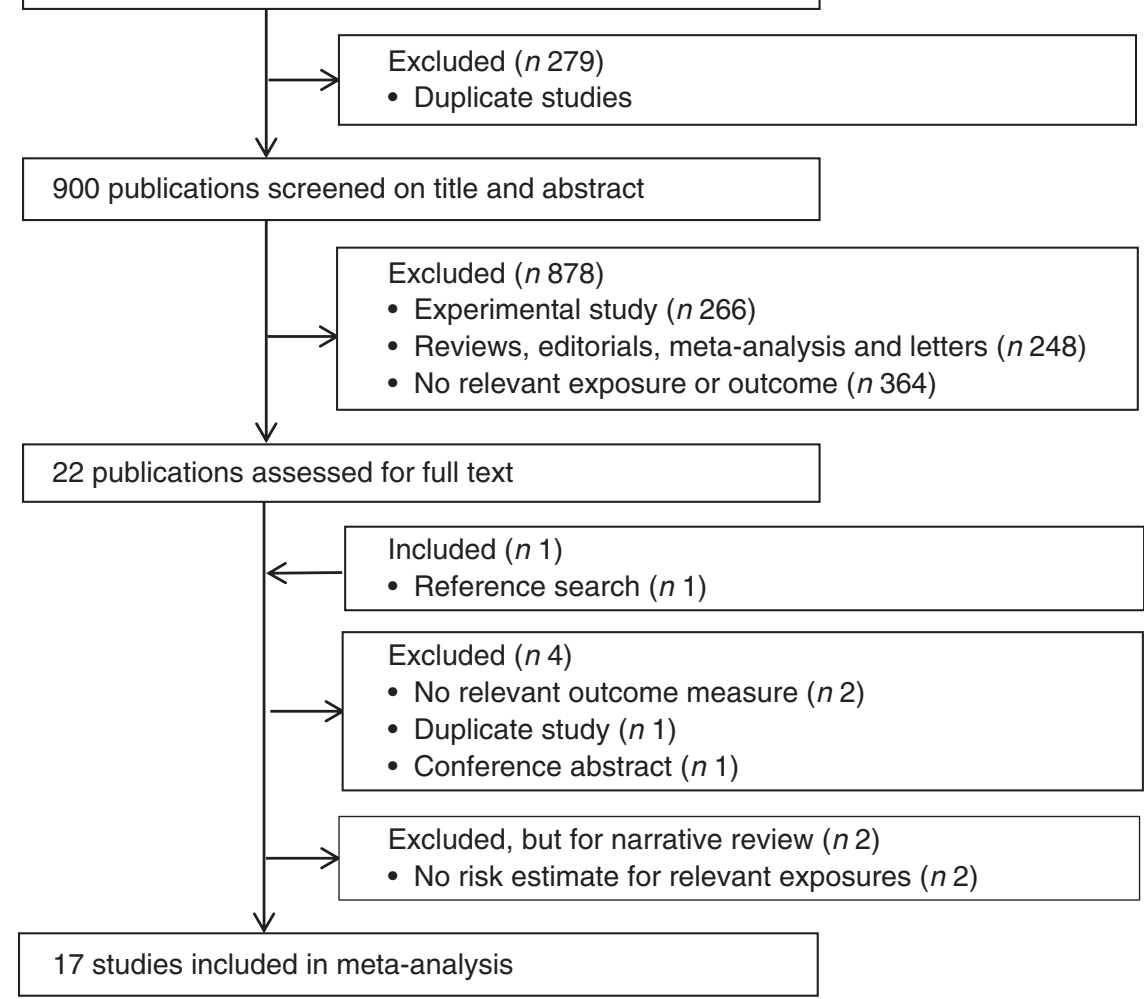

Fig. 1. Flow chart of publication selection. 
isoflavones concentration was skewed. Therefore, seventeen publications were selected for the meta-analysis.

\section{Study characteristics}

The characteristics of the seventeen studies included in the meta-analysis are shown in Table 1, among which there are eight prospective studies (including five cohort studies $^{(21,23,24,26,28)}$, two nested case-control studies ${ }^{(27,36)}$ and one case-cohort study $\left.^{(44)}\right)$ and nine case-control studies ${ }^{(15-20,25,35,47)}$. RR were reported for colorectal or colon cancer in fifteen studies and colorectal adenomas in two studies ${ }^{(15,35)}$. Nine studies were conducted in Europe $\mathrm{E}^{(17,18,20,27,28,35,36,44,47)}$, seven in Asia $^{(15,19,21,23-26)}$ and one in Canada ${ }^{(16)}$. Among the four cohort studies conducted in Asian populations ${ }^{(21,23,24,26)}$, one focused only on colon cancer risk ${ }^{(23)}$ and one included only women ${ }^{(21)}$. A Swedish cohort also included solely women ${ }^{(28)}$. The NOS quality score ranged from 5 to 9 . One study had a score of $5^{(17)}$, one had a score of $6^{(19)}$, three had a score of $7^{(15,18,20)}$, four had a score of $8^{(16,25,35,47)}$ and the remaining eight studies had a score of $9^{(21,23,24,26,27,28,36,44)}$.

\section{Overall phyto-oestrogens}

A total of six cohort ${ }^{(21,23,24,26,28,36)}$ and seven case-control studies $^{(16-20,25,47)}$ were used in the meta-analysis of overall phyto-oestrogen intake and colorectal cancer risk. Two casecontrol studies of colorectal adenoma were added to the metaanalysis for colorectal neoplasm risk ${ }^{(15,35)}$. The pooled RR of colorectal cancer and colorectal neoplasms for the highest $v$. lowest category of overall phyto-oestrogens intake was 0.78 (95\% CI $0.63,0.96)$ and 0.76 (95\% CI 0.63, 0.92), respectively, under a random-effect model. There was significant heterogeneity among studies for both colorectal cancer risk $\left(I^{2}=90 \%\right.$, $\mathrm{pH}<0.01)$ and colorectal neoplasms risk $\left(I^{2}=87 \%, \mathrm{pH}<0.01\right)$. By omitting one study at a time, we identified the Portuguese study by Ravasco et al. ${ }^{(17)}$ as the main source of heterogeneity, which was given a low-quality score of 5 . This study was presented as a cross-sectional study with a case-control design, but the data analysis was performed using a proportional hazards model. Because of the inappropriate data analysis, this study was excluded from further analyses. After excluding the study by Ravasco et al. ${ }^{(17)}$, the pooled RR were 0.84 (95\% CI $0.76,0.92)$ and $0.82(95 \%$ CI $0.75,0.90)$ for colorectal cancer and colorectal neoplasms, respectively (Table 2, Fig. 2). The heterogeneity statistic $I^{2}$ dropped to $32 \%(\mathrm{pH}=0 \cdot 06)$ for colorectal cancer and $29 \%(\mathrm{pH}=0.07)$ for colorectal neoplasms.

Stratified by study design, a significant inverse association with colorectal cancer was observed in case-control studies (pooled RR 0.76; 95\% CI 0.69, 0.84) but not in cohort studies (pooled RR 0.95; 95\% CI 0.85, 1.06) (Table 2, Fig. 2). The results for colorectal cancer were comparable with those for colorectal neoplasms. We did not find substantial differences in associations for colorectal cancer by subgroups in case-control studies. For cohort studies, there were differences between females (pooled RR 0.88; 95\% CI 0.72, 1.08) and males (pooled RR 1.10; $95 \%$ CI 0.68, 1.78), between colon cancer (pooled RR 0.94; $95 \%$ CI $0.78,1.14$ ) and rectal cancer (pooled RR 1.09; 95\% CI 0.79, 1.52), and between Asian (pooled RR 0.93; $95 \%$ CI 0.83, 1.05) and Western studies (pooled RR 1.23; $95 \%$ CI 0.74, 2.05), which were non-significant and based on a limited number of studies. All studies except for two case-control studies included $\mathrm{BMI}^{(16,19)}$ and one included physical activity ${ }^{(16)}$ as adjustment variables. Only two cohort studies accounted for family history of colorectal cancer ${ }^{(21,24)}$ and one accounted for NSAID use ${ }^{(36)}$ whereas four case-control studies adjusted for family history $^{(18,20,25,47)}$ and two adjusted for NSAID use ${ }^{(20,47)}$. In the analyses of colorectal cancer stratified by adjustment factors, the RR was found to be attenuated after adjustment for BMI and physical activity (online Supplementary Table S1). The funnel plot was symmetric and showed no evidence of publication bias $(P=0 \cdot 61$, online Supplementary Fig. S1).

\section{Isoflavones}

The meta-analysis of the association between isoflavones intake and colorectal cancer risk was based on five prospective cohort $^{(21,23,24,26,28)}$ and five case-control studies ${ }^{(16,18-20,25)}$. One additional case-control study ${ }^{(15)}$ was included in the analysis for colorectal neoplasms ${ }^{(15,16,18-21,23-26,28)}$. The pooled RR for the highest $v$. lowest isoflavones intake were 0.85 (95\% CI 0.77 , $0.95)$ and 0.84 (95\% CI $0.76,0.93)$ for colorectal cancer and colorectal neoplasms, respectively (Table 3, online Supplementary Fig. S2). There was evidence of moderate heterogeneity among studies $\left(I^{2}=40 \%, \mathrm{pH}=0.08\right)$. The inverse associations observed for colorectal cancer were statistically significant in case-control studies (pooled RR 0.77; $95 \%$ CI 0.69, 0.85 ) but not in cohort studies (pooled RR 0.94; 95\% CI 0.84, 1.05). We did not observe significant heterogeneity by sex, cancer site or study population in both cohort studies and casecontrol studies.

In the dose-response analyses of dietary isoflavones intake with colorectal cancer risk, five Asian studies ${ }^{(19,21,23,25,26)}$ and four Western studies ${ }^{(9,16,18,28)}$ were included. No evidence of departure from linearity was found for both Asian $(P=0.55)$ and Western studies $(P=0 \cdot 39)$. Inverse associations were identified in both populations, with a pooled RR of 0.92 (95\% CI 0.85 , 1.00 ) for each $20 \mathrm{mg} / \mathrm{d}$ increase in isoflavones intake in Asian populations and 0.98 (95\% CI $0.92,1.05)$ for each $0.1 \mathrm{mg} / \mathrm{d}$ increase in Western populations (Table 4, Fig. 3). Among Asian populations, the inverse association for risk of colorectal neoplasms was statistically significant (pooled RR 0.92; $95 \%$ CI 0.86, 0.97). No evidence of study heterogeneity was observed within both Western studies $\left(I^{2}=29 \%, \mathrm{pH}=0 \cdot 07\right)$ and Asian studies $\left(I^{2}=30 \%, \mathrm{pH}=0 \cdot 20\right)$. In Asian studies, the inverse association was significant in case-control (pooled RR 0.87; 95\% CI 0.79, 0.96) but not cohort studies (pooled RR 0.96; $95 \%$ CI 0.88, 1.05). No substantial differences were observed according to sex and cancer site.

\section{Lignans}

Three studies including two case-control studies ${ }^{(16,20)}$ and one cohort study ${ }^{(28)}$ carried out in Western populations evaluated the association of lignans intake with colorectal cancer risk. The pooled RR for case-control studies indicated a significant association with reduced colorectal cancer risk for the 
Table 1. Characteristics of studies on phyto-oestrogens included in the meta-analysis (Pooled relative risks (RR) and $95 \%$ confidence intervals)

\begin{tabular}{|c|c|c|c|c|c|c|c|c|c|}
\hline \multirow{2}{*}{$\begin{array}{l}\text { Author, country (study } \\
\text { name) (reference) }\end{array}$} & \multirow{2}{*}{$\begin{array}{l}\text { Study } \\
\text { design }\end{array}$} & \multirow{2}{*}{$\begin{array}{l}\text { Sex (number of } \\
\text { subjects (cases/ } \\
\text { non-cases)) }\end{array}$} & \multirow{2}{*}{$\begin{array}{l}\text { Dietary } \\
\text { assessment }\end{array}$} & \multirow{2}{*}{$\begin{array}{l}\text { Type of phyto-oestrogens } \\
\text { (mean/median, } \\
\text { comparison) }\end{array}$} & \multirow[b]{2}{*}{ Outcome } & \multicolumn{2}{|c|}{ Fully adjusted models } & \multirow[b]{2}{*}{ Covariates in fully adjusted models } & \multirow{2}{*}{$\begin{array}{l}\text { Quality } \\
\text { score }\end{array}$} \\
\hline & & & & & & $\mathrm{RR}$ & $95 \% \mathrm{Cl}$ & & \\
\hline $\begin{array}{l}\text { Ravasco et al., } \\
\quad \text { Portugal }^{(17)}\end{array}$ & $\mathrm{HCC}$ & MF $(70 / 70)$ & $\begin{array}{l}\text { FFQ and dietary } \\
\text { history }\end{array}$ & $\begin{array}{l}\text { Isoflavones (NA, Q4 v. Q1: } \\
\quad 20 \text { v. 5) }\end{array}$ & CRC & 0.30 & $0.26,0.34$ & $\begin{array}{l}\text { Age, BMI, family history, smoking, } \\
\text { exercise, comorbidities }\end{array}$ & 5 \\
\hline 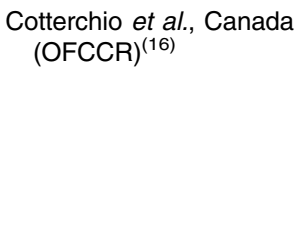 & $\mathrm{PCC}$ & MF (1095/1890) & $\mathrm{FFQ}$ & $\begin{array}{l}\text { Phyto-oestrogens (NA, T3 } \\
\text { v. T1: }>1.34 \mathrm{v} \\
<0.53 \mathrm{mg} / \mathrm{d}) \\
\text { Isoflavones (NA, T3 v. T1: } \\
>1.10 \mathrm{v} .<0.29 \mathrm{mg} / \mathrm{d}) \\
\text { Lignans }(\mathrm{NA}, \mathrm{T} 3 \mathrm{v} . \mathrm{T} 1: \\
>0.26 \mathrm{v} .<0.16 \mathrm{mg} / \mathrm{d})\end{array}$ & $\mathrm{CRC}$ & $\begin{array}{l}0.71 \\
0.71 \\
0.73\end{array}$ & $\begin{array}{l}0.59,0.86 \\
0.58,0.86 \\
0.56,0.94\end{array}$ & $\begin{array}{l}\text { Age, sex, dietary fibre (only for } \\
\text { isoflavones), total energy intake }\end{array}$ & 8 \\
\hline Rossi et al., Italy ${ }^{(18)}$ & $\mathrm{HCC}$ & MF (1953/4154) & $\mathrm{FFQ}$ & $\begin{array}{l}\text { Isoflavones }(25.4 \mu \mathrm{g} / \mathrm{d}, \mathrm{Q} 5 \mathrm{v} \\
\text { Q1: }>33.9 v .<14.4 \mu \mathrm{g} / \mathrm{d})\end{array}$ & $\begin{array}{l}\text { CRC } \\
\text { Colon cancer } \\
\text { Rectal cancer }\end{array}$ & $\begin{array}{l}0.76 \\
0.70 \\
0.81\end{array}$ & $\begin{array}{l}0.63,0.91 \\
0.56,0.87 \\
0.63,1.04\end{array}$ & $\begin{array}{l}\text { Age, sex, study centre, family history } \\
\text { of CRC, education, alcohol } \\
\text { consumption, BMI, occupational } \\
\text { physical activity, energy intake }\end{array}$ & 7 \\
\hline $\begin{array}{l}\text { Kuijsten et al., } \\
\text { The Netherlands } \\
\text { (POLIEP) })^{(35)} \text {; } \\
\text { The Polyp Study }\end{array}$ & $\mathrm{HCC}$ & MF $(532 / 503)$ & LC/MS, plasma & $\begin{array}{l}\text { Enterolactone }(11.6 \mathrm{nmol} / \mathrm{l} \\
\text { Q4 } \mathrm{v} . \mathrm{Q} 1:>26.3 \mathrm{v} . \\
<4.6 \mathrm{nmol} / \mathrm{l}) \\
\text { Per } 1 \log _{10} \text { unit with an } \\
\text { increment of } \log (39.1)\end{array}$ & CRA & $\begin{array}{l}0.66 \\
0.75\end{array}$ & $\begin{array}{l}0.44,0.98 \\
0.52,1.08\end{array}$ & $\begin{array}{l}\text { Age, sex, alcohol, NSAID use, BMI, } \\
\text { physical activity, smoking, family } \\
\text { history of CRC, antibiotic use, } \\
\text { indication for endoscopy }\end{array}$ & 8 \\
\hline $\begin{array}{l}\text { Theodoratou et al., UK } \\
\text { (Study of Colorectal }^{(47)} \\
\text { Cancer in Scotland) }\end{array}$ & $\mathrm{PCC}$ & $\mathrm{M} / \mathrm{F}(1456 / 1456)$ & $\mathrm{FFQ}$ & $\begin{array}{l}\text { Phyto-oestrogens }(595 \mu \mathrm{g} / \mathrm{d} \text {, } \\
\text { Q4 v. Q1: > 858 v. } \\
\quad<403 \mu \mathrm{g} / \mathrm{d})\end{array}$ & $\mathrm{CRC}$ & 0.90 & $0 \cdot 72,1 \cdot 13$ & $\begin{array}{l}\text { Family history of CRC, total energy } \\
\text { intake, total fibre intake, alcohol } \\
\text { intake, NSAID intake, smoking, } \\
\text { BMI, and physical activity }\end{array}$ & 8 \\
\hline $\begin{array}{l}\text { Oba et al., Japan } \\
\quad \text { (Takayama Study) }^{(23)}\end{array}$ & cs & $\begin{array}{l}M(111 / 13894) \\
F(102 / 16327)\end{array}$ & $\mathrm{FFQ}$ & $\begin{array}{l}\text { Isoflavones }(38.0 \mathrm{mg} / \mathrm{d}, \mathrm{T} 3 \\
v . \mathrm{T} 1: 59.6 \mathrm{v} .<22.5 \mathrm{mg} / \mathrm{d}) \\
\text { Isoflavones }(35.8 \mathrm{mg} / \mathrm{d}, \mathrm{T} 3 \mathrm{v} \text {. } \\
\text { T1: }>54.6 \mathrm{v} .<21.7 \mathrm{mg} / \mathrm{d})\end{array}$ & Colon cancer & $\begin{array}{l}1.47 \\
0.73\end{array}$ & $\begin{array}{l}0.90,2.40 \\
0.44,1 \cdot 18\end{array}$ & $\begin{array}{l}\text { Age, height, alcohol intake, smoking } \\
\text { status, BMI, physical exercise, } \\
\text { coffee intake and use of hormone- } \\
\text { replacement therapy }\end{array}$ & 9 \\
\hline $\begin{array}{l}\text { Akhter et al., Japan } \\
\text { (JPHC-based } \\
\text { prospective study })^{(26)}\end{array}$ & CS & $\begin{array}{l}\text { M (528/39 069) } \\
F(358 / 43994)\end{array}$ & $\mathrm{FFQ}$ & $\begin{array}{l}\text { Isoflavones (NA, Q4 v. Q1: } \\
50.4 \text { v. } 9.1 \mathrm{mg} / \mathrm{d}) \\
\text { Isoflavones (NA, Q4 v. Q1: } \\
49.7 \text { v. } 9.1 \mathrm{mg} / \mathrm{d})\end{array}$ & CRC & $\begin{array}{l}0.89 \\
1.07\end{array}$ & $\begin{array}{l}0.67,1.17 \\
0.78,1.47\end{array}$ & $\begin{array}{l}\text { Age, public health centre area, } \\
\text { diabetes history, BMI, physical } \\
\text { activity, smoking, alcohol, intake } \\
\text { of vitamin D, meat, fish, dairy } \\
\text { products, fruit, and vegetable. } \\
\text { Menopausal status, current use of } \\
\text { female hormones }\end{array}$ & 9 \\
\hline
\end{tabular}


Table 1. Continued

\begin{tabular}{|c|c|c|c|c|c|c|c|c|c|}
\hline \multirow{2}{*}{$\begin{array}{l}\text { Author, country (study } \\
\text { name) (reference) }\end{array}$} & \multirow{2}{*}{$\begin{array}{l}\text { Study } \\
\text { design }\end{array}$} & \multirow{2}{*}{$\begin{array}{l}\text { Sex (number of } \\
\text { subjects (cases/ } \\
\text { non-cases)) }\end{array}$} & \multirow{2}{*}{$\begin{array}{l}\text { Dietary } \\
\text { assessment }\end{array}$} & \multirow{2}{*}{$\begin{array}{l}\text { Type of phyto-oestrogens } \\
\text { (mean/median, } \\
\text { comparison) }\end{array}$} & \multirow[b]{2}{*}{ Outcome } & \multicolumn{2}{|c|}{ Fully adjusted models } & \multirow[b]{2}{*}{ Covariates in fully adjusted models } & \multirow{2}{*}{$\begin{array}{l}\text { Quality } \\
\text { score }\end{array}$} \\
\hline & & & & & & $\mathrm{RR}$ & $95 \% \mathrm{Cl}$ & & \\
\hline $\begin{array}{l}\text { Butler et al., Singapore } \\
\text { (Singapore Chinese } \\
\text { Health study) })^{(24)}\end{array}$ & CS & MF (961/61 321) & $\mathrm{FFQ}$ & $\begin{array}{l}\text { Isoflavones (9.8 mg/ } \\
1000 \mathrm{kcal}, \mathrm{Q} 4 \text { v. Q1:) }\end{array}$ & CRC & 0.95 & $0.79,1.13$ & $\begin{array}{l}\text { Age, sex, dialect group, interview } \\
\text { year, diabetes at baseline, } \\
\text { smoking, BMI, alcohol intake, } \\
\text { education, physical activity, family } \\
\text { history of CRC, and daily energy } \\
\text { intake }\end{array}$ & 9 \\
\hline $\begin{array}{l}\text { Ward et al., UK (EPIC- } \\
\text { NORFOLK) }\end{array}$ & NCC & MF (221/889) & LC/MS, serum & $\begin{array}{l}\text { Enterolactone }(5 \cdot 2 \mu \mathrm{g} / \mathrm{ml}) \\
\text { Lignans }(5 \cdot 7 \mu \mathrm{g} / \mathrm{ml}) \\
\text { Isoflavones }(9 \cdot 3 \mu \mathrm{g} / \mathrm{ml}) \\
\text { Per doubling the exposure } \\
\quad \text { level }\end{array}$ & CRC & $\begin{array}{l}1.02 \\
1.03 \\
1.01\end{array}$ & $\begin{array}{l}0.95,1.10 \\
0.94,1.12 \\
0.94,1.08\end{array}$ & $\begin{array}{l}\text { Age, sex, height, weight, average } \\
\text { intake of fibre and } \mathrm{Ca}\end{array}$ & 9 \\
\hline $\begin{array}{l}\text { Kuijsten et al., } \\
\text { The Netherlands } \\
\text { (Monitoring Project on } \\
\text { Cardiovascular } \\
\text { Diseases Risk } \\
\text { Factor) }^{(36)}\end{array}$ & NCC & MF (160/387) & LC/MS, plasma & $\begin{array}{l}\text { Enterolactone } \\
\quad(8.8 \mathrm{nmol} / /, \mathrm{Q} 4 \mathrm{v.Q} 1: \\
\quad \geq 18.75 \mathrm{v} .4 .11 \mathrm{nmol} / \mathrm{l}) \\
\text { Per } 1 \log _{10} \text { unit with an } \\
\text { increment of } \log (14.61)\end{array}$ & CRC & $\begin{array}{l}1.70 \\
1.56\end{array}$ & $\begin{array}{l}0.88,3.27 \\
0.89,2.72\end{array}$ & $\begin{array}{l}\text { BMI, alcohol and smoking } \\
\text { consumption, duration of } \\
\text { smoking, physical activity, aspirin } \\
\text { use, education. Intakes of energy, } \\
\text { fibre, } \mathrm{Ca} \text {, tea, wine, meat, fish, } \\
\text { fruits, vegetables and whole-grain } \\
\text { bread }\end{array}$ & 9 \\
\hline $\begin{array}{l}\text { Yang et al., China } \\
\text { (Shanghai Women's } \\
\text { Health) }\end{array}$ & CS & $F(321 / 68412)$ & $\mathrm{FFQ}$ & $\begin{array}{l}\text { Isoflavones }(30.8 \mathrm{mg} / \mathrm{d}, \mathrm{T} 3 \\
v . \mathrm{T} 1:>34.8 \mathrm{v} \\
<20.9 \mathrm{mg} / \mathrm{d})\end{array}$ & CRC & 0.76 & $0.56,1.01$ & $\begin{array}{l}\text { Age, education, household income, } \\
\text { physical activity, BMI, menopausal } \\
\text { status, family history of CRC, total } \\
\text { energy intake, and average } \\
\text { intakes of fruits, vegetables, red } \\
\text { meat, non-soya Ca, non-soya } \\
\text { fibre, and non-soya folic acid }\end{array}$ & 9 \\
\hline $\begin{array}{l}\text { Akhter et al., Japan } \\
\text { (CAST) }^{(15)}\end{array}$ & $\mathrm{HCC}$ & $\begin{array}{l}\text { MF }(721 / 697) \\
\text { M (492/460) } \\
\text { F (229/237) }\end{array}$ & $\mathrm{FFQ}$ & $\begin{array}{l}\text { Isoflavones }(39.73 \mathrm{mg} / \mathrm{d} \\
\text { Q4 } \mathrm{v} . \mathrm{Q} 1: \geq 62.4 \mathrm{v} \\
<24.8 \mathrm{mg} / \mathrm{d})\end{array}$ & $\begin{array}{l}\text { CRA } \\
\text { Proximal colon } \\
\text { adenoma } \\
\text { Distal colon adenoma } \\
\text { Rectum adenoma } \\
\text { CRA } \\
\text { CRA }\end{array}$ & $\begin{array}{l}0.70 \\
0.69 \\
\\
0.71 \\
0.68 \\
0.74 \\
0.49\end{array}$ & $\begin{array}{l}0.51,0.96 \\
0.47,1.00 \\
0.46,1.09 \\
0.36,1.31 \\
0.50,1.09 \\
0.27,0.90\end{array}$ & $\begin{array}{l}\text { Age, screening, family history of } \\
\text { CRC, cigarette smoking, alcohol } \\
\text { consumption, BMI, physical } \\
\text { activity, supplement use, NSAID } \\
\text { use (age at menarche, } \\
\text { menopausal status and current } \\
\text { use of female hormones for } \\
\text { female only) }\end{array}$ & 7 \\
\hline $\begin{array}{l}\text { Johnsen et al., Denmark } \\
\text { (Diet, Cancer and } \\
\text { Health) }\end{array}$ & Case-cohort & $\begin{array}{l}M(213 / 207) \\
F(168 / 163)\end{array}$ & TR/FIA, plasma & $\begin{array}{l}\text { Enterolactone }(24 \mathrm{nmol} / \mathrm{l}) \\
\text { Enterolactone }(34 \mathrm{nmol} / \mathrm{l}) \\
\text { Per doubling the } \\
\quad \text { enterolactone level }\end{array}$ & $\begin{array}{l}\text { Colon cancer } \\
\text { Rectal cancer } \\
\text { Colon cancer } \\
\text { Rectal cancer }\end{array}$ & $\begin{array}{l}1 \cdot 19 \\
1.74 \\
0.73 \\
0 \cdot 83\end{array}$ & $\begin{array}{l}0.93,1.51 \\
1.25,2.44 \\
0.56,0.94 \\
0.57,1.20\end{array}$ & $\begin{array}{l}\text { BMI, present smoking, bowel } \\
\text { movements per week, intake of } \\
\text { alcohol and whole grain products }\end{array}$ & 9 \\
\hline $\begin{array}{l}\text { Budhathoki et al., Japan } \\
\text { (Fukuoka Colorectal } \\
\text { Cancer Study) }\end{array}$ & PCC & $\begin{array}{l}\text { MF (816/815) } \\
M(488 / 504) \\
F(328 / 311)\end{array}$ & Dietary interview & $\begin{array}{l}\text { Isoflavones }(37.2 \mathrm{mg} / \mathrm{d} \text {, Q5 } \\
\text { v. Q1: } 74.4 v .15 .5 \mathrm{mg} / \mathrm{d})\end{array}$ & CRC & $\begin{array}{l}0.76 \\
0.68 \\
0.94\end{array}$ & $\begin{array}{l}0.53,1.09 \\
0.42,1.10 \\
0.52,1.71\end{array}$ & $\begin{array}{l}\text { Sex, age, resident area, parental } \\
\text { CRC, smoking, alcohol use, BMI, } \\
\text { type of job, and leisure time } \\
\text { physical activity, intakes of Ca and } \\
n-3 \text { PUFA }\end{array}$ & 8 \\
\hline
\end{tabular}




\section{NS British Journal of Nutrition}

Table 1. Continued

\begin{tabular}{|c|c|c|c|c|c|c|c|c|c|}
\hline \multirow{2}{*}{$\begin{array}{l}\text { Author, country (study } \\
\text { name) (reference) }\end{array}$} & \multirow{2}{*}{$\begin{array}{l}\text { Study } \\
\text { design }\end{array}$} & \multirow{2}{*}{$\begin{array}{l}\text { Sex (number of } \\
\text { subjects (cases/ } \\
\text { non-cases)) }\end{array}$} & \multirow{2}{*}{$\begin{array}{l}\text { Dietary } \\
\text { assessment }\end{array}$} & \multirow{2}{*}{$\begin{array}{l}\text { Type of phyto-oestrogens } \\
\text { (mean/median, } \\
\text { comparison) }\end{array}$} & \multirow[b]{2}{*}{ Outcome } & \multicolumn{2}{|c|}{ Fully adjusted models } & \multirow[b]{2}{*}{ Covariates in fully adjusted models } & \multirow{2}{*}{$\begin{array}{l}\text { Quality } \\
\text { score }\end{array}$} \\
\hline & & & & & & $\mathrm{RR}$ & $95 \% \mathrm{Cl}$ & & \\
\hline $\begin{array}{l}\text { Zamora-Ros et al., Spain } \\
\text { (Bellvitge Colorectal } \\
\text { Cancer Study) })^{(20)}\end{array}$ & $\mathrm{HCC}$ & MF (421/401) & FFQ & $\begin{array}{l}\text { Phyto-oestrogens }(1.0 \mathrm{mg} / \mathrm{d} \text {, } \\
\text { Q4 v. Q1: }>0.74 \mathrm{v} . \\
0.39 \mathrm{mg} / 4184 \mathrm{~kJ} \mathrm{~d} \\
(0.39 \mathrm{mg} / 1000 \mathrm{kcal} \mathrm{d})) \\
\text { Isoflavones }(0.2 \mathrm{mg} / \mathrm{d}, \mathrm{Q} 4 \mathrm{v} \text {. } \\
\text { Q1: }>0.17 \mathrm{v} .<0.07 \mathrm{mg} / \\
1000 \mathrm{kcal} \mathrm{d}) \\
\text { Lignans }(0.7 \mathrm{mg} / \mathrm{d}, \mathrm{Q} 4 \mathrm{v} . \\
\text { Q1: }>0.50 \mathrm{v} .<0.27 \mathrm{mg} / \\
1000 \mathrm{kcal} \mathrm{d})\end{array}$ & $\begin{array}{l}\text { CRC } \\
\text { Colon cancer } \\
\text { Rectal cancer } \\
\text { CRC } \\
\text { Colon cancer } \\
\text { Rectal cancer } \\
\text { CRC } \\
\text { Colon cancer } \\
\text { Rectal cancer }\end{array}$ & $\begin{array}{l}0.53 \\
0.55 \\
0.41 \\
\\
1.25 \\
1.50 \\
0.83 \\
0.59 \\
0.55 \\
0.62\end{array}$ & $\begin{array}{l}0.30,0.93 \\
0.28,1.06 \\
0.19,0.91 \\
0.82,1.88 \\
0.93,2.41 \\
0.47,1.47 \\
0.34,0.99 \\
0.28,1.06 \\
0.30,1.29\end{array}$ & $\begin{array}{l}\text { Sex, age, BMI, energy intake, } \\
\text { alcohol consumption, fibre intake, } \\
\text { red and processed meat intake, } \\
\text { tobacco consumption, physical } \\
\text { activity, regular drugs (aspirin, } \\
\text { NSAID), and family history of } \\
\text { CRC }\end{array}$ & 7 \\
\hline Shin et al., Korea ${ }^{(19)}$ & $\mathrm{HCC}$ & F (298/894) & FFQ & $\begin{array}{l}\text { Isoflavones (NA, Q4 v. Q1: } \\
\quad \geq 22.35 \mathrm{v} .<8.08 \mathrm{mg} / \mathrm{d})\end{array}$ & $\begin{array}{l}\text { CRC } \\
\text { Proximal colon } \\
\text { cancer } \\
\text { Distal colon cancer } \\
\text { Rectal cancer } \\
\text { CRC } \\
\text { Proximal colon } \\
\text { cancer } \\
\text { Distal colon cancer } \\
\text { Rectal cancer }\end{array}$ & $\begin{array}{l}0.71 \\
1.29 \\
\\
0.34 \\
0.88 \\
0.78 \\
0.89 \\
\\
1 \cdot 16 \\
0.60\end{array}$ & $\begin{array}{l}0.52,0.97 \\
0.69,2.42 \\
\\
0.20,0.59 \\
0.56,1.32 \\
0.50,1.23 \\
0.32,1.11 \\
\\
0.60,2.26 \\
0.32,1.11\end{array}$ & $\begin{array}{l}\text { Age, education, alcohol } \\
\text { consumption, regular exercise }\end{array}$ & 6 \\
\hline $\begin{array}{l}\text { Hedelin et al., Sweden } \\
\quad(\mathrm{WLH})^{(28)}\end{array}$ & CS & $F(206 / 46268)$ & FFQ & $\begin{array}{l}\text { Isoflavones (NA, Q4 v. Q1: } \\
23 v .0 .6 \mu \mathrm{g} /(\mathrm{d} \times \mathrm{MJ})) \\
\text { Lignans }(\mathrm{NA}, \mathrm{Q} 4 \mathrm{v.} \mathrm{Q1} \text { : } \\
\quad 497 v .236 \mu \mathrm{g} /(\mathrm{d} \times \mathrm{MJ}))\end{array}$ & $\begin{array}{l}\text { CRC } \\
\text { Colon cancer } \\
\text { Rectal cancer } \\
\text { CRC } \\
\text { Colon cancer } \\
\text { Rectal cancer }\end{array}$ & $\begin{array}{l}1.06 \\
0.95 \\
1.08 \\
1.00 \\
0.91 \\
1.10\end{array}$ & $\begin{array}{l}0.59,1.29 \\
0.57,1.58 \\
0.56,2.08 \\
0.64,1.57 \\
0.55,1.51 \\
0.58,2.07\end{array}$ & $\begin{array}{l}\text { Age, energy intake, BMI, education, } \\
\text { smoking, physical activities, } \\
\text { dietary intake of processed meat, } \\
\text { alcohol, SFA, vitamin D, } \\
\text { vegetables, fruits, fish and fibre, } \\
\text { individual phyto-oestrogens }\end{array}$ & 9 \\
\hline
\end{tabular}

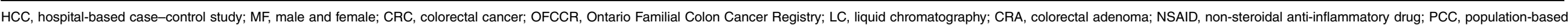

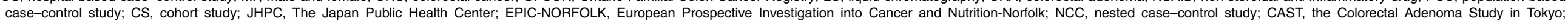
TR/FIA, time-resolved fluoroimmuno-assay; WLH, Women's Lifestyle and Health cohort study. 
highest $v$. lowest category (pooled RR 0.70; 95\% CI 0.56, 0.89). However, no association was found in the cohort study. No study heterogeneity was detected $\left(I^{2}=0, \mathrm{pH}=0 \cdot 30\right)$ (Table 3 , online Supplementary Fig. S3). In the dose-response analyses of these three studies, significant departure from linearity was detected $(P<0 \cdot 01)$. A non-linear relationship was observed, with an inverse association between dietary lignans intake and colorectal cancer at low-to-moderate intakes and no further reduction in risk at higher intakes $>2.5 \mathrm{mg} / \mathrm{d}$ (Fig. 4).

The dose-response analysis for circulating enterolactone concentrations was based on three prospective studies $(27,36,44)$ for colorectal cancer risk and one additional case-control study for colorectal neoplasms risk ${ }^{(35)}$, also solely in Western populations. No association was observed of the risk of either colorectal cancer (pooled RR 1.04; 95\% CI 0.98, 1.10) or colorectal neoplasms (pooled RR 1.00; $95 \%$ CI 0.96, 1.05) with per doubling of blood enterolactone concentration (Table 4, online Supplementary Fig. S4). However, a $22 \%$ increased risk of rectal cancer (95\% CI 1.04, 1.43) was observed, whereas no association was seen for colon cancer (pooled RR 1.00; 95\% CI $0 \cdot 89,1 \cdot 12)$.

\section{Discussion}

The meta-analysis suggests that a greater phyto-oestrogen intake may be associated with a reduced risk of colorectal cancer and colorectal neoplasms. The inverse association was, however, statistically significant in case-control studies and not in cohort studies. Dose-response analysis of isoflavones indicated an $8 \%$ lower risk of colorectal neoplasms per $20 \mathrm{mg} / \mathrm{d}$ increase in Asian populations. For lignans, a non-linear inverse association with colorectal cancer risk was found for dietary lignans intake, whereas there was no association for circulating enterolactone concentrations.

For overall phyto-oestrogens, there was evidence of moderate heterogeneity in the association with colorectal cancer. First, the study design could be a potential source of heterogeneity, as heterogeneity was detected between subgroups but not within subgroups by study design. Case-control studies are considered prone to recall bias, that is, cancer patients are more likely to recall a potential cancer-related diet. In pooled analyses, four of six Western studies were case-control studies, whereas four of the six Asian studies were cohort studies. Therefore, recall bias may partly explain the more prominent effects in case-control studies and Western populations. Heterogeneity could also be partly due to the fact that the pooled RR was derived from estimates of different phytooestrogen subgroups, including dietary phyto-oestrogens, dietary isoflavones, dietary lignans and blood enterolactone concentrations. Considering the different results for isoflavones and enterolactone, it appears warranted to discuss the association with colorectal cancer for isoflavones and lignans separately. The symmetric funnel plot suggested no evidence of small study effects, such as publication bias.

The association of isoflavones intake with a $16 \%$ reduced risk of colorectal cancer is consistent with a recent metaanalysis $^{(29)}$, which found an $24 \%$ reduced colorectal cancer risk 


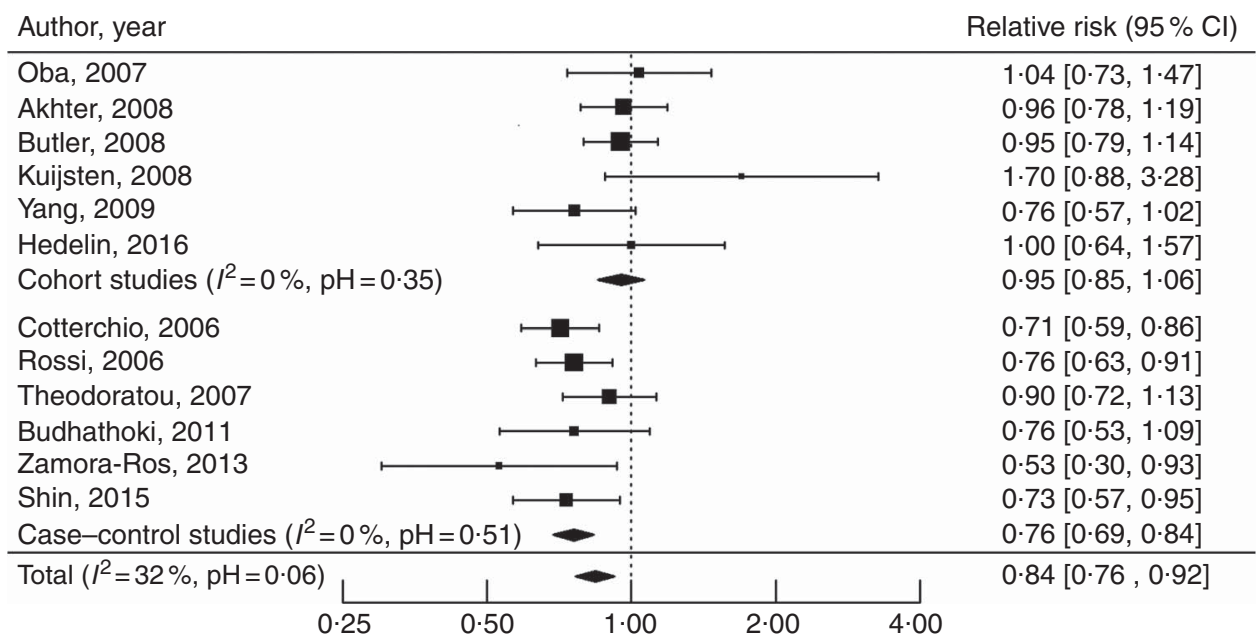

Fig. 2. Pooled relative risk of colorectal cancer for highest $v$. lowest phyto-oestrogens; results from a random-effect meta-analysis.

Table 3. Meta-analyses of the association (highest $v$. lowest categories) of dietary isoflavones and dietary lignans with colorectal cancer by selected subgroups

(Pooled relative risks (RR) and $95 \%$ confidence intervals)

\begin{tabular}{|c|c|c|c|c|c|c|c|c|c|c|}
\hline \multirow[b]{2}{*}{ Group } & \multicolumn{5}{|c|}{ Colorectal cancer only } & \multicolumn{5}{|c|}{ Colorectal cancer and adenoma } \\
\hline & No. of studies & Pooled RR & $95 \% \mathrm{Cl}$ & $P(\%)$ & $\mathrm{pH}^{*}$ & No. of studies & Pooled RR & $95 \% \mathrm{Cl}$ & $P(\%)$ & $\mathrm{pH}^{*}$ \\
\hline \multicolumn{11}{|c|}{ Dietary isoflavones $\dagger$} \\
\hline Overall & 10 & 0.85 & $0.77,0.95$ & 41 & 0.08 & 11 & 0.84 & $0.76,0.93$ & 37 & 0.09 \\
\hline Cohort & 5 & 0.94 & $0.84,1.05$ & 0 & 0.62 & 5 & 0.94 & $0.84,1.05$ & 0 & 0.62 \\
\hline \multicolumn{11}{|l|}{ Sex } \\
\hline Male & 2 & 1.09 & $0.68,1.78$ & 67 & 0.08 & 2 & 1.09 & $0.68,1.78$ & 67 & 0.08 \\
\hline Female & 3 & 0.88 & $0.72,1.08$ & 23 & 0.31 & 3 & 0.88 & $0.72,1.08$ & 23 & 0.31 \\
\hline \multicolumn{11}{|l|}{ Cancer site } \\
\hline Colon & 3 & 0.94 & $0.78,1 \cdot 12$ & 0 & 0.93 & 3 & 0.94 & $0.78,1.14$ & 0 & 0.81 \\
\hline Rectal & 2 & 1.09 & $0.78,1.51$ & 0 & 0.98 & 2 & 1.08 & $0.78,1.51$ & 0 & 0.98 \\
\hline \multicolumn{11}{|c|}{ Study population } \\
\hline Asian & 4 & 0.93 & $0.83,1.05$ & 0 & 0.50 & 4 & 0.93 & $0.83,1.05$ & 0 & 0.50 \\
\hline Western & 1 & 1.06 & $0.68,1.65$ & 0 & 1.00 & 1 & 1.06 & $0.68,1.65$ & 0 & 1.00 \\
\hline Case-control & 5 & 0.77 & $0.69,0.85$ & 0 & $0 \cdot 19$ & 6 & 0.76 & $0.68,0.84$ & 0 & 0.26 \\
\hline \multicolumn{11}{|l|}{ Sex } \\
\hline Male & 2 & 0.70 & $0.54,0.91$ & 0 & 0.88 & 3 & 0.71 & $0.57,0.89$ & 0 & 0.96 \\
\hline Female & 2 & 0.83 & $0.58,1.20$ & 0 & 0.62 & 3 & 0.72 & $0.53,1.00$ & 7 & 0.29 \\
\hline \multicolumn{11}{|l|}{ Cancer site } \\
\hline Colon & 3 & 0.89 & $0.58,1.37$ & 82 & 0.02 & 4 & 0.82 & $0.61,1 \cdot 10$ & 73 & 0.03 \\
\hline Rectal & 3 & 0.80 & $0.66,0.97$ & 0 & 0.98 & 4 & 0.79 & $0.66,0.95$ & 0 & 0.96 \\
\hline \multicolumn{11}{|c|}{ Study population } \\
\hline Asian & 2 & 0.74 & $0.60,0.91$ & 0 & 0.87 & 3 & 0.73 & $0.61,0.87$ & 0 & 0.94 \\
\hline Western & 3 & 0.83 & $0.62,1.11$ & 76 & 0.05 & 3 & 0.83 & $0.62,1.11$ & 76 & 0.05 \\
\hline \multicolumn{11}{|l|}{ Dietary lignans } \\
\hline Overall & 3 & 0.76 & $0.61,0.93$ & 0 & 0.30 & 3 & 0.76 & $0.61,0.93$ & 0 & 0.30 \\
\hline Cohort & 1 & 1.00 & $0.64,1.57$ & 0 & 1.00 & 1 & 1.00 & $0.64,1.57$ & 0 & 1.00 \\
\hline Case-control & 2 & 0.70 & $0.56,0.89$ & 0 & 0.48 & 2 & $0 \cdot 70$ & $0.56,0.89$ & 0 & 0.48 \\
\hline
\end{tabular}

* Heterogeneity within subgroup.

† Under random-effect models.

$\ddagger$ Under fixed-effect model.

for highest $v$. lowest categories based on eight studies. However, Tse \& Eslick ${ }^{(29)}$ did not include three recent studies ${ }^{(19,20,28)}$ and did not trim the cross-sectional study that caused asymmetry of the funnel plot $^{(17)}$, which may have led to overestimation of risk reduction. We found significant heterogeneity by study design. On the basis of cohort studies, the inverse association for dietary isoflavones was weaker and not statistically significant. The lack of significance could be due to insufficient power considering the weak association, but this could also indicate that there is no association. Any real association is likely to be closer in magnitude to that based on the cohort studies. An earlier meta-analysis already discussed the absence of association for three Asian cohort studies $^{(21,23,26)}$ in contrast to inverse associations found in case-control studies in Western countries ${ }^{(13)}$. 
Table 4. Dose-response analysis of the association of dietary isoflavones and circulating enterolactone with colorectal cancer risk under random-effect models (Pooled relative risks (RR) and 95\% confidence intervals)

\begin{tabular}{|c|c|c|c|c|c|c|c|c|c|c|}
\hline \multirow[b]{2}{*}{ Group } & \multicolumn{5}{|c|}{ Colorectal cancer only } & \multicolumn{5}{|c|}{ Colorectal cancer and adenoma } \\
\hline & No. of studies & Pooled RR & $95 \% \mathrm{Cl}$ & $P^{2}(\%)$ & $\mathrm{pH}^{*}$ & No. of studies & Pooled RR & $95 \% \mathrm{Cl}$ & $P(\%)$ & $\mathrm{pH}^{*}$ \\
\hline \multicolumn{11}{|l|}{ Dietary Isoflavones } \\
\hline \multicolumn{11}{|l|}{ Asian population† } \\
\hline Overall & 5 & 0.92 & $0.85,1.00$ & 30 & 0.20 & 6 & 0.92 & $0.86,0.97$ & 15 & 0.27 \\
\hline \multicolumn{11}{|l|}{ Sex } \\
\hline Male & 4 & 0.93 & $0.80,1.07$ & 60 & 0.08 & 4 & 0.92 & $0.86,0.99$ & 0 & 0.14 \\
\hline Female & 5 & 0.92 & $0.82,1.04$ & 31 & 0.28 & 6 & 0.90 & $0.81,1.00$ & 34 & 0.26 \\
\hline \multicolumn{11}{|l|}{ Cancer site } \\
\hline Colon & 3 & 0.95 & $0.86,1.04$ & 1 & 0.20 & 4 & 0.92 & $0.86,0.98$ & 0 & 0.26 \\
\hline Rectal & 2 & 0.94 & $0.74,1.19$ & 51 & 0.15 & 3 & 0.92 & $0.79,1.07$ & 30 & 0.25 \\
\hline \multicolumn{11}{|l|}{ Study design } \\
\hline Cohort & 3 & 0.96 & $0.88,1.05$ & 11 & 0.26 & 3 & 0.96 & $0.88,1.05$ & 11 & 0.26 \\
\hline Case-control & 2 & 0.87 & $0.79,0.96$ & 0 & 0.36 & 3 & 0.88 & $0.82,0.95$ & 0 & 0.61 \\
\hline \multicolumn{11}{|c|}{ Western population $\ddagger$} \\
\hline Overall & 4 & 0.98 & $0.92,1.05$ & 29 & 0.07 & 4 & 0.98 & $0.92,1.05$ & 29 & 0.07 \\
\hline Cohort & 1 & 0.98 & $0.76,1.26$ & 0 & 1.00 & 1 & 0.98 & $0.76,1.26$ & 0 & 1.00 \\
\hline Case-control & 3 & 0.93 & $0 \cdot 75,1 \cdot 16$ & 90 & 0.03 & 3 & 0.93 & $0.75,1.16$ & 90 & 0.03 \\
\hline \multicolumn{11}{|l|}{ Blood enterolactone§ } \\
\hline Overall & $3 \|$ & 1.04 & $0.98,1 \cdot 10$ & 0 & 0.50 & 4 & 1.00 & $0.96,1.05$ & 44 & $0 \cdot 15$ \\
\hline \multicolumn{11}{|l|}{ Sex } \\
\hline Male & 2 & $1 \cdot 15$ & $0.85,1.58$ & 83 & 0.02 & 2 & $1 \cdot 15$ & $0.85,1.58$ & 83 & 0.02 \\
\hline Female & 2 & 0.99 & $0.59,1.66$ & 91 & 0.00 & 2 & 0.99 & $0.59,1.66$ & 91 & 0.00 \\
\hline \multicolumn{11}{|l|}{ Cancer site } \\
\hline Colon & 2 & 1.00 & $0.89,1 \cdot 12$ & 0 & 0.46 & 2 & 1.00 & $0.89,1.12$ & 0 & 0.46 \\
\hline Rectal & 2 & 1.22 & $1.04,1.43$ & 6 & 0.81 & 2 & 1.22 & $1.04,1.43$ & 6 & 0.81 \\
\hline
\end{tabular}

* Heterogeneity within subgroup.

† RR presented for per $20 \mathrm{mg} / \mathrm{d}$ increase in isoflavones intake.

$\ddagger$ RR presented for per $0.1 \mathrm{mg} / \mathrm{d}$ increase in isoflavones intake.

$\S$ RR presented for per doubling the enterolactone concentration.

II RR based on three cohort studies.

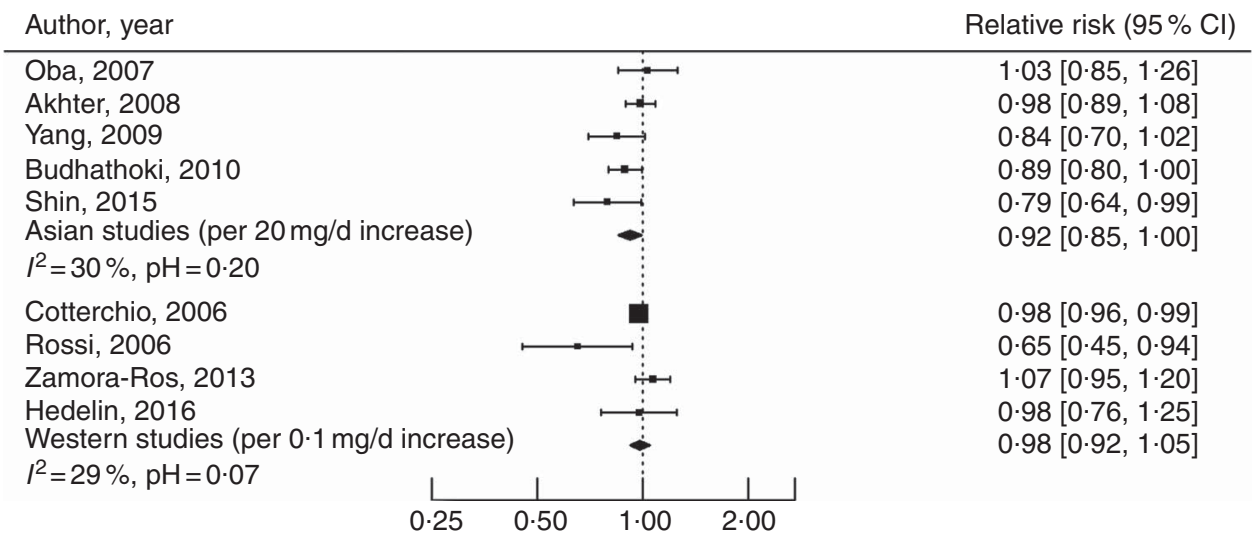

Fig. 3. Pooled relative risk of colorectal cancer for isoflavones intake; results from a random-effect dose-response meta-analysis.

Isoflavones consumption varies greatly between Asian and Western populations. Asian populations have an average isoflavones consumption of $>30 \mathrm{mg} / \mathrm{d}$, whereas Western populations consume $<1 \mathrm{mg} / \mathrm{d}^{(9)}$. Apart from differences in the amount of isoflavones intake, processing of isoflavonecontaining foods could also differ between Asian and Western populations and in turn influence the bioavailability of isoflavones ${ }^{(12)}$. In Asia, the major source of isoflavones is soyabean-fermented products such as tofu and tempeh, which contain the aglycones form of isoflavones. However, in Western diets, isoflavones come from cooked soyabeans, soya milk and texturised vegetable proteins mainly in the form of glucosides. The aglycones form of isoflavones can be absorbed by the intestine directly, whereas glucoside forms need to be hydrolysed by $\beta$-glucosidases to aglycone forms in the jejunum. This large geographic difference in isoflavones consumption levels and processing approach justified the stratification of the dose-response analysis by ethnicity of the study population. Given lower levels and less variability of isoflavones intake across Western populations, the dose-response analysis did not yield a significant inverse relationship as in the Asian population. However, there were only four Western studies $(16,18,20,28)$ 


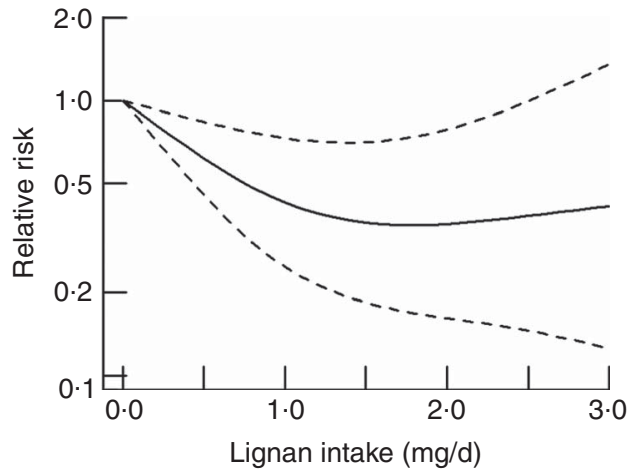

Fig. 4. Pooled dose-response association between dietary lignans and colorectal cancer risk; results from a random-effect dose-response metaanalysis.

that could be included in the meta-analysis, of which one was a prospective study ${ }^{(28)}$ that found no association. In a further prospective study in the UK that could not be included, neither dietary intake ${ }^{(22)}$ nor serum levels of isoflavones ${ }^{(27)}$ were found to be associated with colorectal cancer risk. Thus, large-scale, prospective investigations, particularly in Western populations, are warranted to understand the possible protective role of isoflavones in the development of colorectal cancer.

Inconsistent findings for dietary lignans intake and blood enterolactone concentrations with respect to colorectal cancer risk were observed. A non-linear, dose-response association between dietary lignans and colorectal cancer risk was detected in our study, whereas no significant association was detected for per doubling increase of blood enterolactone levels. It may be because of several reasons. First, this could be explained by the study design, as the results regarding dietary lignans were based mainly on case-control studies and that regarding enterolactone were based solely on cohort studies. Second, lignans intake was estimated from FFQ representing long-term consumption, whereas circulating enterolactone concentration captures recent short-term status. Although FFQ measurement may be subject to recall bias, long-term status might be more relevant for cancer development. Moreover, dietary lignans only account for a moderate amount of variation in circulating enterolactone concentrations ${ }^{(49)}$. Plant lignans are converted by gut microflora into the bioactive metabolites enterolactone and enterodiol. As the binding sites for bioactive lignan metabolites are limited, the protective effect of lignans might reach a plateau when the level of lignan metabolites achieves a certain threshold. Thus, a non-linear association between dietary lignans and colorectal cancer risk as observed in our study seems biologically plausible. Enterolactone is the predominant metabolite, and therefore is the preferred biomarker to measure exposure to the bioactive component of lignans ${ }^{(50,51)}$. However, only one ${ }^{(44)}$ of the four included studies accounted for the effect of antibiotics, which influences the activity of gut microflora, and therefore is a strong determinant of enterolactone and enterodiol concentration, and found that this strengthened the association with colorectal cancer risk. Because of the small number of studies, which are all from European countries, including only one cohort study on dietary lignans intake, the current evidence is too limited to draw conclusions regarding lignans.

Overall, the associations of isoflavones and lignans with colorectal neoplasms are consistent with those for colorectal cancer. Colorectal adenoma is an early step in the development of colorectal cancer, and therefore is an informative end point for studying colorectal carcinogenesis. Studies have shown that isoflavones and lignans are associated with a reduced risk of both colorectal adenoma and its recurrence ${ }^{(15,35,52,53)}$. These consistent findings suggest that phyto-oestrogens might have a chemo-preventive effect already in the initial stages of colorectal carcinogenesis. This is in line with results from animal studies, where phyto-oestrogens are associated with a reduction in a number of early carcinogenesis-related markers such as formation of aberrant crypt foci, size and number of $\operatorname{adenomas}^{(54-57)}$.

Our study is the first to quantify the dose-response relationship of the association of colorectal cancer risk with dietary isoflavones and lignans, and to systematically evaluate the association of colorectal cancer risk with the lignans intake, lignan biomarkers and overall phyto-oestrogens. Previous metaanalyses solely compared the highest $v$. lowest category to evaluate the association between isoflavones and colorectal cancer risk. With the dose-response meta-analysis, it was possible to assess linearity of the relationships and visualise the non-linear associations for different exposure levels. To include all available studies for more accurate estimates, we converted differing exposure measurements into the same unit and pooled the trend estimate for colorectal cancer in each study. This allows a comprehensive view on the association between phyto-oestrogen and colorectal cancer risk based on current available evidence.

Observational studies have their inherent limitation of residual confounding, which will remain in the pooled analysis. A higher intake of phyto-oestrogens may be associated with a healthier lifestyle ${ }^{(9)}$, which may influence colorectal cancer risk independently. In the stratified analyses by adjusted factors, these factors were not found to influence the overall association substantially. Although some residual confounding may exist, the inverse associations observed are unlikely to be explained solely by healthy lifestyle, as almost all studies adjusted for the most relevant lifestyle factors. Another possible limitation is that different phyto-oestrogen subgroups were investigated, and different measurement methods were used in the included studies, which may lead to inconsistent results. Although some of this variation may be real, the use of various nutrient databases may have led to differences in estimated dietary phytooestrogen intake between the studies. As discussed above, FFQ is subject to recall bias whereas blood biomarkers could only reflect short-term exposure and are influenced by gut microflora, BMI, smoking and constipation ${ }^{(50,51)}$. Therefore, different determinants of various phyto-oestrogen subgroups might result in varying associations between phyto-oestrogens and colorectal cancer. We used the random-effect model to account for the variation and heterogeneity between studies. Applying RR for the highest $v$. lowest category in the overall meta-analysis made it possible to pool studies with different assessment methods and different categorisations of exposure. There was 
effect heterogeneity according to the study design for the association between phyto-oestrogens and colorectal cancer risk. Inverse associations for phyto-oestrogens overall and by subgroup were significant in case-controls studies but not in cohort studies. Furthermore, there was limited power for precise risk estimation of the association of different phyto-oestrogen subgroups with colorectal cancer because of the overall small number of cohort studies, especially for lignans, for which there was only one cohort study evaluating this relationship.

Several mechanisms may explain the potential anticarcinogenic effects of phyto-oestrogens ${ }^{(2)}$. Similar to oestrogen, phyto-oestrogens can bind to oestrogen receptors and possess a higher binding affinity to oestrogen receptor $\beta$ (ESR2). ESR2 is the predominantly expressed oestrogen receptor in normal colon mucosa, and progressively decreases in the pathological mucosa paralleling the grade and the stage of colorectal cancer ${ }^{(58)}$. In the presence of ESR2, phyto-oestrogens exhibit anti-proliferative effects at a lower and more physiological concentration $(0 \cdot 1-10 \mu \mathrm{mol} / \mathrm{l})^{(59)}$. In addition, both animal studies and a randomised controlled trial in human subjects showed that dietary phyto-oestrogens can increase the expression levels of ESR2 in the colonic mucosa ${ }^{(60-62)}$. ESR2 expression could also inhibit colonic tumour growth in the xenograft mouse model ${ }^{(63)}$.

In summary, the currently available epidemiological studies did not provide sufficient evidence to draw a rigorous conclusion regarding an effect of phyto-oestrogens on colorectal cancer risk. The association of isoflavones with reduced risk of colorectal cancer was statistically significant only in casecontrol studies but not in cohort studies, which are less prone to recall and selection bias. Evidence of an association between dietary lignans or their active metabolites and colorectal cancer risk is limited, and cohort studies evaluating dietary lignans intake are lacking. It is presently unknown whether an effect of phyto-oestrogen could differ by ESR2 expression of the tumour. In view of the importance of colorectal cancer in cancer incidence and mortality in the general population, further prospective studies are warranted to clarify the association between different phyto-oestrogens and colorectal cancer risk and assess possible differential effects by ESR2 expression.

\section{Acknowledgements}

The work was supported by the German Federal Ministry of Education and Research (grant numbers 01ER0814 and 01ER0815) and the National Cancer Institute, National Institutes of Health, US Department of Health and Human Services (grant numbers U01 CA137088 and R01 CA164930).

R. J., A. B. performed the literature search; R. J., A. H. analysed the data; R. J. drafted the article; A. B., A. R. and J. C.-C. critically reviewed the manuscript; all the authors read and approved the final manuscript.

The authors disclose no potential conflicts of interest.

\section{Supplementary material}

For supplementary material/s referred to in this article, please visit https://doi.org/10.1017/S0007114516004360

\section{References}

1. Ferlay JSI, Ervik M, Dikshit R, et al. (2013) Cancer incidence and mortality worldwide: IARC CancerBase no. 11. http:// globocan.iarc.fr (accessed January 2016).

2. Pampaloni CMB, Bartolini E, Tonelli F, et al. (2013) Phytoestrogens and colon cancer. In Soybean-Bio-Active Compounds, pp. 75-96 [HA El-Shemy, editor]. InTech.

3. Kuiper GG, Carlsson B, Grandien K, et al. (1997) Comparison of the ligand binding specificity and transcript tissue distribution of estrogen receptors alpha and beta. Endocrinology 138, 863-870.

4. Kuiper GG, Lemmen JG, Carlsson B, et al. (1998) Interaction of estrogenic chemicals and phytoestrogens with estrogen receptor beta. Endocrinology 139, 4252-4263.

5. Barzi A, Lenz AM, Labonte MJ, et al. (2013) Molecular pathways: estrogen pathway in colorectal cancer. Clin Cancer Res 19, 5842-5848.

6. Cornwell T, Cohick W \& Raskin I (2004) Dietary phytoestrogens and health. Phytochemistry 65, 995-1016.

7. Thompson LU, Boucher BA, Liu Z, et al. (2006) Phytoestrogen content of foods consumed in Canada, including isoflavones, lignans, and coumestan. Nutr Cancer 54, 184-201.

8. Kuhnle GG, Dell'Aquila C, Aspinall SM, et al. (2008) Phytoestrogen content of foods of animal origin: dairy products, eggs, meat, fish, and seafood. J Agric Food Chem 56, 10099-10104.

9. Zamora-Ros R, Knaze V, Lujan-Barroso L, et al. (2012) Dietary intakes and food sources of phytoestrogens in the European Prospective Investigation into Cancer and Nutrition (EPIC) 24-hour dietary recall cohort. Eur J Clin Nutr 66 , 932-941.

10. Valentin-Blasini L, Sadowski MA, Walden $\mathrm{D}$, et al. (2005) Urinary phytoestrogen concentrations in the U.S. population (1999-2000). J Expo Anal Environ Epidemiol 15, 509-523.

11. Rowland I, Faughnan M, Hoey L, et al. (2003) Bioavailability of phyto-oestrogens. Br J Nutr 89, Suppl. 1, S45-S58.

12. Zubik L \& Meydani M (2003) Bioavailability of soybean isoflavones from aglycone and glucoside forms in American women. Am J Clin Nutr 77, 1459-1465.

13. Yan L, Spitznagel EL \& Bosland MC (2010) Soy consumption and colorectal cancer risk in humans: a meta-analysis. Cancer Epidemiol Biomarkers Prev 19, 148-158.

14. Woo HD \& Kim J (2013) Dietary flavonoid intake and risk of stomach and colorectal cancer. World J Gastroenterol 19, 1011-1019

15. Akhter M, Iwasaki M, Yamaji T, et al. (2009) Dietary isoflavone and the risk of colorectal adenoma: a case-control study in Japan. Br J Cancer 100, 1812-1816.

16. Cotterchio M, Boucher BA, Manno M, et al. (2006) Dietary phytoestrogen intake is associated with reduced colorectal cancer risk. J Nutr 136, 3046-3053.

17. Ravasco P, Monteiro-Grillo I, Marques Vidal P, et al. (2005) Nutritional risks and colorectal cancer in a Portuguese population. Nutr Hosp 20, 165-172.

18. Rossi M, Negri E, Talamini R, et al. (2006) Flavonoids and colorectal cancer in Italy. Cancer Epidemiol Biomarkers Prev 15, 1555-1558.

19. Shin A, Lee J, Lee J, et al. (2015) Isoflavone and soyfood intake and colorectal cancer risk: a case-control study in Korea. PLOS ONE 10, e0143228.

20. Zamora-Ros R, Not C, Guinó E, et al. (2013) Association between habitual dietary flavonoid and lignan intake and colorectal cancer in a Spanish case-control study (the Bellvitge Colorectal Cancer Study). Cancer Causes Control 24, 549-557. 
21. Yang G, Shu XO, Li H, et al. (2009) Prospective cohort study of soy food intake and colorectal cancer risk in women. $\mathrm{Am} \mathrm{J}$ Clin Nutr 89, 577-583.

22. Ward HA, Kuhnle GGC, Mulligan AA, et al. (2010) Breast, colorectal, and prostate cancer risk in the European Prospective Investigation into Cancer and Nutrition-Norfolk in relation to phytoestrogen intake derived from an improved database. Am J Clin Nutr 91, 440-448.

23. Oba S, Nagata C, Shimizu N, et al. (2007) Soy product consumption and the risk of colon cancer: a prospective study in Takayama, Japan. Nutr Cancer 57, 151-157.

24. Butler LM, Wang R, Koh WP, et al. (2008) Prospective study of dietary patterns and colorectal cancer among Singapore Chinese. Br J Cancer 99, 1511-1516.

25. Budhathoki S, Joshi AM, Ohnaka K, et al. (2011) Soy food and isoflavone intake and colorectal cancer risk: the Fukuoka Colorectal Cancer Study. Scand I Gastroenterol 46, 165-172.

26. Akhter M, Inoue M, Kurahashi N, et al. (2008) Dietary soy and isoflavone intake and risk of colorectal cancer in the Japan public health center-based prospective study. Cancer Epidemiol Biomarkers Prev 17, 2128-2135.

27. Ward H, Chapelais G, Kuhnle GGC, et al. (2008) Lack of prospective associations between plasma and urinary phytoestrogens and risk of prostate or colorectal cancer in the European Prospective into Cancer-Norfolk study. Cancer Epidemiol Biomarkers Prev 17, 2891-2894.

28. Hedelin M, Lof M, Sandin S, et al. (2016) Prospective study of dietary phytoestrogen intake and the risk of colorectal cancer. Nutr Cancer 68, 388-395.

29. Tse G \& Eslick GD (2016) Soy and isoflavone consumption and risk of gastrointestinal cancer: a systematic review and meta-analysis. Eur J Nutr 55, 63-73.

30. Moher D, Liberati A, Tetzlaff J, et al. (2009) Preferred reporting items for systematic reviews and meta-analyses: the PRISMA statement. PLoS Med 6, e1000097.

31. Wells GA, Shea B, O'Connell D, Peterson J, et al. (2000) The Newcastle-Ottawa Scale (NOS) for assessing the quality of nonrandomised studies in meta-analyses. http://www.ohri. $\mathrm{ca} /$ programs/clinical_epidemiology/oxford.asp (accessed January 2016).

32. Greenland S \& Longnecker MP (1992) Methods for trend estimation from summarized dose-response data, with applications to meta-analysis. Am J Epidemiol 135, 1301-1309.

33. Orsini N, Li R, Wolk A, et al. (2012) Meta-analysis for linear and nonlinear dose-response relations: examples, an evaluation of approximations, and software. Am I Epidemiol 175, 66-73.

34. Harrell FE Jr., Lee KL \& Pollock BG (1988) Regression models in clinical studies: determining relationships between predictors and response. J Natl Cancer Inst $\mathbf{8 0}$, 1198-1202.

35. Kuijsten A, Arts ICW, Hollman PCH, et al. (2006) Plasma enterolignans are associated with lower colorectal adenoma risk. Cancer Epidemiol Biomarkers Prev 15, 1132-1136.

36. Kuijsten A, Hollman PCH, Boshuizen HC, et al. (2008) Plasma enterolignan concentrations and colorectal cancer risk in a nested case-control study. Am J Epidemiol 167, 734-742.

37. Higgins JP \& Thompson SG (2002) Quantifying heterogeneity in a meta-analysis. Stat Med 21, 1539-1558.

38. Hedges LV \& Pigott TD (2001) The power of statistical tests in meta-analysis. Psychol Methods 6, 203-217.

39. Egger M, Davey Smith G, Schneider M, et al. (1997) Bias in meta-analysis detected by a simple, graphical test. BMJ $\mathbf{3 1 5}$ 629-634.

40. Viechtbauer W (2010) Conducting meta-analyses in R with the metafor package. J Stat Softw 36, 1-48.
41. Crippa A \& Orsini N (2016) Multivariate dose-response metaanalysis: the dosresmeta R package. J Stat Softw 72, 1-15.

42. Cutler GJ, Nettleton JA, Ross JA, et al. (2008) Dietary flavonoid intake and risk of cancer in postmenopausal women: The Iowa Women's Health Study. Int J Cancer $\mathbf{1 2 3}$, 664-671.

43. Honma N, Yamamoto K, Ohnaka K, et al. (2013) Estrogen receptor-beta gene polymorphism and colorectal cancer risk: effect modified by body mass index and isoflavone intake. Int J Cancer 132, 951-958.

44. Johnsen NF, Olsen A, Thomsen BL, et al. (2010) Plasma enterolactone and risk of colon and rectal cancer in a casecohort study of Danish men and women. Cancer Causes Control 21, 153-162.

45. Li L, Zhang M \& Holman CD (2013) Population versus hospital controls in the assessment of dietary intake of isoflavone for case-control studies on cancers in China. Nutr Cancer $\mathbf{6 5}$, 390-397.

46. Rossi M, Bosetti C, Negri E, et al. (2010) Flavonoids, proanthocyanidins, and cancer risk: a network of case-control studies from Italy. Nutr Cancer 62, 871-877.

47. Theodoratou E, Kyle J, Cetnarskyj R, et al. (2007) Dietary flavonoids and the risk of colorectal cancer. Cancer Epidemiol Biomarkers Prev 16, 684-693.

48. Butler LM, Koh WP, Wang R, et al. (2010) Soy increases risk of rectal cancer among Singapore Chinese women. Cancer Res 70, 8 Suppl., Abstract 2815.

49. Milder IEJ, Kuijsten A, Arts ICW, et al. (2007) Relation between plasma enterodiol and enterolactone and dietary intake of lignans in a Dutch endoscopy-based population. J Nutr 137, 1266-1271.

50. Kilkkinen A, Stumpf K, Pietinen P, et al. (2001) Determinants of serum enterolactone concentration. Am J Clin Nutr $\mathbf{7 3}$, $1094-1100$

51. Horner NK, Kristal AR, Prunty J, et al. (2002) Dietary determinants of plasma enterolactone. Cancer Epidemiol Biomarkers Prev 11, 121-126.

52. Bobe G, Sansbury LB, Albert PS, et al. (2008) Dietary flavonoids and colorectal adenoma recurrence in the Polyp Prevention Trial. Cancer Epidemiol Biomarkers Prev 17, 1344-1353.

53. Bobe G, Murphy G, Albert PS, et al. (2012) Dietary lignan and proanthocyanidin consumption and colorectal adenoma recurrence in the Polyp Prevention Trial. Int J Cancer 130, 1649-1659.

54. Danbara N, Yuri T, Tsujita-Kyutoku M, et al. (2005) Enterolactone induces apoptosis and inhibits growth of Colo 201 human colon cancer cells both in vitro and in vivo. Anticancer Res 25, 2269-2276.

55. Zhang Y, Li Q, Zhou D, et al. (2013) Genistein, a soya isoflavone, prevents azoxymethane-induced up-regulation of WNT/beta-catenin signalling and reduces colon pre-neoplasia in rats. Br J Nutr 109, 33-42.

56. Thiagarajan DG, Bennink MR, Bourquin LD, et al. (1998) Prevention of precancerous colonic lesions in rats by soy flakes, soy flour, genistein, and calcium. Am J Clin Nutr $\mathbf{6 8}$, 1394s-1399s.

57. Javid SH, Moran AE, Carothers AM, et al. (2005) Modulation of tumor formation and intestinal cell migration by estrogens in the $\operatorname{Apc}(\mathrm{Min} /+)$ mouse model of colorectal cancer. Carcinogenesis 26, 587-595.

58. Caiazza F, Ryan EJ, Doherty G, et al. (2015) Estrogen receptors and their implications in colorectal carcinogenesis. Front Oncol 5, 19.

59. Totta P, Acconcia F, Virgili F, et al. (2005) Daidzein-sulfate metabolites affect transcriptional and antiproliferative 
activities of estrogen receptor-beta in cultured human cancer cells. J Nutr 135, 2687-2693.

60. Principi M, Di Leo A, Pricci M, et al. (2013) Phytoestrogens/ insoluble fibers and colonic estrogen receptor beta: randomized, double-blind, placebo-controlled study. World J Gastroenterol 19, 4325-4333.

61. Raju J, Bielecki A, Caldwell D, et al. (2009) Soy isoflavones modulate azoxymethane-induced rat colon carcinogenesis exposed pre- and postnatally and inhibit growth of DLD-1 human colon adenocarcinoma cells by increasing the expression of estrogen receptor-beta. J Nutr 139, 474-481.

62. Barone M, Tanzi S, Lofano K, et al. (2010) Dietary-induced ERbeta upregulation counteracts intestinal neoplasia development in intact male ApcMin/+ mice. Carcinogenesis 31, 269-274.

63. Hartman J, Edvardsson K, Lindberg K, et al. (2009) Tumor repressive functions of estrogen receptor beta in SW480 colon cancer cells. Cancer Res 69, 6100-6106. 